ARTICLE

https://doi.org/10.1038/s41467-019-11649-0

\title{
Periodicity of molecular clusters based on symmetry-adapted orbital model
}

\author{
Takamasa Tsukamoto (1) 1,2,4, Naoki Haruta (1) 1,2,3,4, Tetsuya Kambe (1) 1,2, Akiyoshi Kuzume ${ }^{1,2}$ \& \\ Kimihisa Yamamoto ${ }^{1,2}$
}

The periodic table has always contributed to the discovery of a number of elements. Is there no such principle for larger-scale substances than atoms? Many stable substances such as clusters have been predicted based on the jellium model, which usually assumes that their structures are approximately spherical. The jellium model is effective to explain subglobular clusters such as icosahedral clusters. To broaden the scope of this model, we propose the symmetry-adapted orbital model, which explicitly takes into account the level splittings of the electronic orbitals due to lower structural symmetries. This refinement indicates the possibility of an abundance of stable clusters with various shapes that obey a certain periodicity. Many existing substances are also governed by the same rule. Consequently, all substances with the same symmetry can be unified into a periodic framework in analogy to the periodic table of elements, which will act as a useful compass to find missing substances.

\footnotetext{
${ }^{1}$ Institute of Innovative Research, Tokyo Institute of Technology, Yokohama 226-8503, Japan. ${ }^{2}$ ERATO, JST, Kawaguchi, Saitama 332-0012, Japan. ${ }^{3}$ Fukui Institute for Fundamental Chemistry, Kyoto University, Sakyou-ku, Kyoto 606-8103, Japan. ${ }^{4}$ These authors contributed equally:Takamasa Tsukamoto, Naoki Haruta. Correspondence and requests for materials should be addressed to K.Y. (email: yamamoto@res.titech.ac.jp)
} 
n 1869, Mendeleev proposed the periodic table of the elements, which has long been the foundation of the natural sciences ${ }^{1}$. The periodic table has contributed to the discovery of a number of the elements that obey a certain periodic rule. Thereafter, much attention has been paid to higher-order substances that consist of the elements, including molecules, clusters, nanoparticles, and bulk substances. Although such substances have tremendous potential to be functional materials, it remains a difficult problem to predict and design unknown substances among the infinite combinations of constitutive elements. As with Mendeleev's periodic table, such a periodic table for higher-order substances would be considerably valuable for a wide range of materials science. Is there no principle to govern larger-scale substances?

Recently, Tomalia and Khanna ${ }^{2}$ pointed out the possibility of nanoperiodicity in higher-order substances typified by superatoms that function as building blocks of higher-order architectures $^{3-5}$ as with atoms that form molecules. It is known that some particularly stable clusters exhibit properties that resemble specific chemical elements in the periodic table, e.g., $\left[\mathrm{Al}_{13}\right]^{-}$ and $\left[\mathrm{Al}_{7} \mathrm{C}\right]^{-6,7}$. Such clusters are referred to as superatoms, which have electronic structures similar to those of atoms $s^{2,6-12}$. The superatoms have been predicted on the basis of the jellium model ${ }^{1,13}$, which usually assumes that these structures have an approximately spherical symmetry. As described by the jellium model, molecular orbitals generated by valence electrons in highly symmetric clusters have shapes just like atomic orbitals. Accordingly, a cluster will be stable when the number of valence electrons coincides with a closed-shell structure based on the jellium model. This spherical approximation is applicable especially for subglobular $I_{h}$ symmetric clusters. The initially discovered superatom was the $\left[\mathrm{Al}_{13}\right]^{-}$cluster with $I_{h}$ symmetry ${ }^{6}$.

A theoretical framework that describes a broad range of substances is necessary to obtain a universal law. The spherical jellium model is a candidate for such a theory; however, most clusters do not have quasi-spherical $I_{h}$ symmetry ${ }^{14,15}$, because their atomicities are inappropriate for the construction of $I_{h}$ symmetry structures. Even if they have the icosahedral atomicities, many of the clusters exhibit Jahn-Teller or pseudo Jahn-Teller instability ${ }^{16}$ due to electronic reasons. On the other hand, particularly stable clusters without $I_{h}$ symmetry have also been found, such as $\mathrm{Au}_{20}$ with $T_{d}$ symmetry ${ }^{8}$. In such clusters, the validity of the spherical jellium model decreases relatively due to the split and shift of the superatomic orbital levels. Therefore, it is strongly desired to establish a more sophisticated method to find an abundance of stable clusters. We have focused on the violation of the spherical jellium model to extend the theory for the prediction of clusters. In the present study, we demonstrate that various stable clusters can be correctly predicted by considering their structural symmetry, in addition to the number of valence electrons and the number of constitutive atoms. This is the essence of our theory, the symmetry-adapted orbital model (SAO model) to reveal the hidden periodicity behind largerscale substances.

\section{Results}

Symmetry-adapted orbital model. The SAO model is introduced as follows. Detailed formulation is given in the Supplementary Notes 1 and 2. Herein we discuss three types of structural symmetries based on Platonic solids: $I_{h}, O_{h}$, and $T_{d}$. Superatomic orbitals derived from the spherical jellium model typically split under these structural symmetries. As shown in Table 1, the splitting pattern is completely determined within the point-group

\begin{tabular}{|c|c|c|c|}
\hline Spherical & $I_{h}$ & $o_{h}$ & $\mathbf{T}_{\boldsymbol{d}}$ \\
\hline$S$ & $\overline{A_{g}}$ & $A_{1 g}$ & $A_{1}$ \\
\hline$P$ & $T_{1 u}^{g}$ & $T_{1 u}^{19}$ & $T_{2}$ \\
\hline$D$ & $H_{g}$ & $E_{g} \oplus T_{2 g}$ & $E \oplus T_{2}$ \\
\hline$F$ & $T_{2 u} \oplus G_{u}$ & $A_{2 u} \oplus T_{1 u} \oplus T_{2 u}$ & $A_{1} \oplus T_{1} \oplus T_{2}$ \\
\hline G & $G_{g} \oplus H_{g}$ & $A_{1 g} \oplus E_{g} \oplus T_{1 g} \oplus T_{2 g}$ & $A_{1} \oplus E \oplus T_{1} \oplus T_{2}$ \\
\hline$H$ & $T_{1 u} \oplus T_{2 u} \oplus H_{u}$ & $E_{u} \oplus 2 T_{1 u} \oplus T_{2 u}$ & $E \oplus T_{1} \oplus 2 T_{2}$ \\
\hline
\end{tabular}

theory ${ }^{17,18}$. In the case of $I_{h}$ symmetry, the $F$ and $G$ orbitals split into $T_{2 u}$ and $G_{u}$ orbitals, as well as $G_{g}$ and $H_{g}$ orbitals, respectively. For the $O_{h}$ symmetry, the $D$ orbitals split into $E_{g}$ and $T_{2 g}$ orbitals. The $F$ and $G$ orbitals split in a more complicated way. For the $T_{d}$ symmetry, the splitting pattern is almost the same as that for $O_{h}$.

However, the order of orbital levels is not determined by the point-group theory ${ }^{17,18}$, but is dependent on the degree of level splittings. According to our quantum chemical calculations, the order of the orbital levels generally obeys a certain law with respect to each structural symmetry (Fig. 1). The reason is as follows. Let us take the spherical jellium model as the unperturbed system, as described in the Supplementary Notes 1 and 2 . The perturbation of the nuclear charge distribution gives rise to the shift and split of the jellium orbital levels. According to the first-order perturbation theory, the jellium orbitals that overlap the nuclear charge distribution are selectively stabilized, depending on each structural symmetry, as compared with the spherical jellium model ${ }^{19}$. When the split orbitals are filled with a suitable number of valence electrons, structural symmetry is maintained without any Jahn-Teller distortion ${ }^{16}$. According to this model, the magic numbers, or the number of valence electrons required for full occupation of the split orbitals, are dependent on the structural symmetries. The magic numbers for each symmetry are listed in Table 2 (see also Fig. 1). It should also be noted that atomicity must be appropriate for the construction of each symmetric structure. The present SAO model is quite simple but more effective than expected, as revealed later with diverse examples.

First-principles calculations. The SAO model was verified by comparison with the density functional theory (DFT) calculations, using the $T_{d}$-type clusters as an example. Figure 2 shows that the molecular orbitals of the $T_{d}$-type clusters can be ascribed to the split superatomic orbitals. According to the SAO model, in the case of four-atom clusters $\left(E_{4}\right)$, only the elements having 2,4, and 5 valence electrons fill the split orbitals. For 10 -atom and 20 -atom clusters $\left(E_{10}\right.$ and $\left.E_{20}\right)$, elements with one valence electron are also allowed. DFT calculations were performed for $E_{4}$ clusters comprised of typical elements in the same period, as shown in Fig. 3. The elements $\mathrm{Cd}, \mathrm{In}, \mathrm{Sn}, \mathrm{Sb}$, and Te have 2, 3, 4, 5, and 6 valence electrons, respectively. It was confirmed that the $E_{4}$ clusters are stable with $T_{d}$ symmetry only for the elements with 2,4 , and 5 valence electrons. These elements coincide with full occupations of the SAOs which correspond to $1 P\left(T_{2}\right), 1 D\left(T_{2}\right)$, and $1 D(E)$, respectively. No imaginary frequencies were observed in any of these clusters. On the other hand, the $\mathrm{In}_{4}$ and $\mathrm{Te}_{4}$ clusters are distorted due to the Jahn-Teller effect ${ }^{16}$, which originates from the partial fillings of the degenerate SAOs. However, suitable charge control allows these clusters to conserve their $T_{d}$ symmetry, such as $\left[\mathrm{In}_{4}\right]^{2+}$ and $\left[\mathrm{Te}_{4}\right]^{4+}$ 


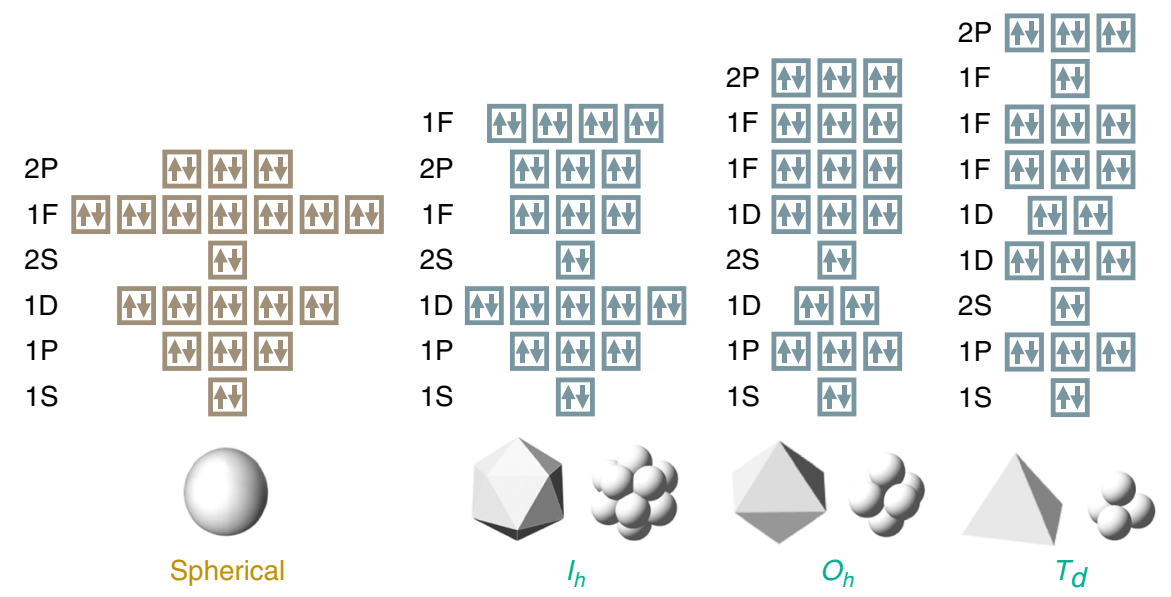

Fig. 1 Electronic configurations based on the symmetry-adapted orbital (SAO) models. The order of the orbital levels generally obeys this rule, although many valence electron systems have strong interelectronic interactions and distorted structures, often changing the order of orbital levels partially

Table 2 Magic numbers of valence electrons based on the SAO models

\section{Magic number of valence electrons}

\begin{tabular}{|c|c|c|c|c|c|c|c|c|c|}
\hline Spherical & $\begin{array}{l}2 \\
(1 S)^{2}\end{array}$ & $\begin{array}{l}8 \\
(1 P)^{6}\end{array}$ & & $\begin{array}{l}18 \\
(1 D)^{10}\end{array}$ & $\begin{array}{l}20 \\
(2 S)^{2}\end{array}$ & & & $\begin{array}{l}34 \\
(1 F)^{14}\end{array}$ & $\begin{array}{l}40 \\
(2 P)^{6}\end{array}$ \\
\hline$T_{d}$ & $\begin{array}{l}2 \\
(1 S)^{2}\end{array}$ & $\begin{array}{l}8 \\
(1 P)^{6}\end{array}$ & $\begin{array}{l}10 \\
(2 S)^{2}\end{array}$ & $\begin{array}{l}16 \\
\left(1 D^{a}\right)^{6}\end{array}$ & $\begin{array}{l}20 \\
\left(1 D^{a}\right)^{4}\end{array}$ & $\begin{array}{l}26 \\
\left(1 F^{a}\right)^{6}\end{array}$ & $\begin{array}{l}32 \\
\left(1 F^{a}\right)^{6}\end{array}$ & $\begin{array}{l}34 \\
\left(1 F^{a}\right)^{2}\end{array}$ & $\begin{array}{l}40 \\
(2 P)^{6}\end{array}$ \\
\hline
\end{tabular}

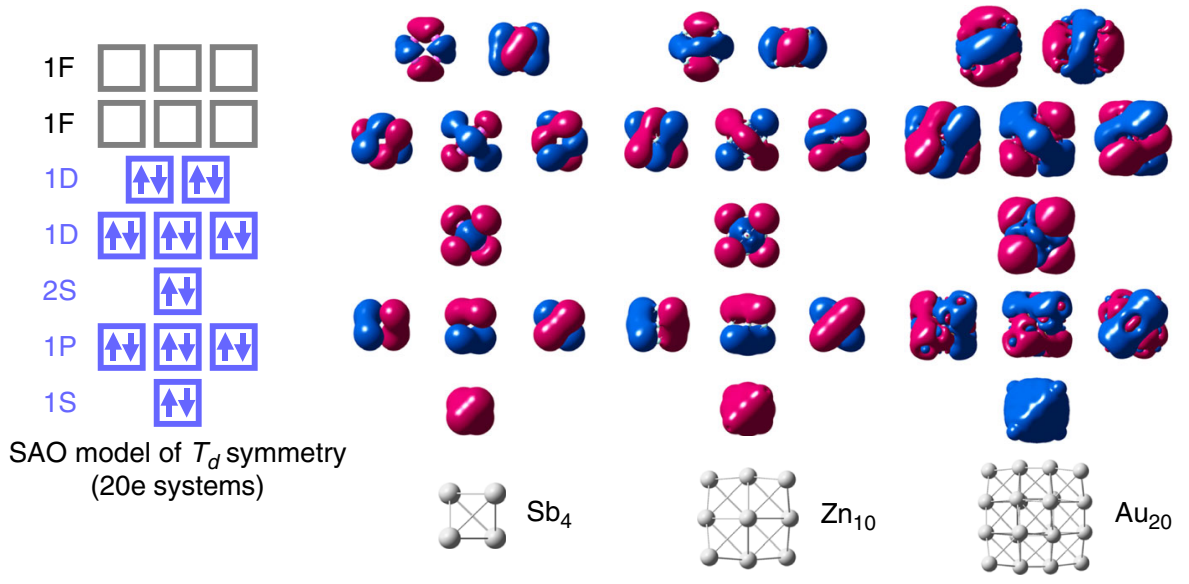

Fig. 2 Molecular orbitals of $T_{d}$-type clusters. They can be ascribed to the split superatomic orbitals, depending on the orbital angular momenta

(Supplementary Figs. 1 and 2). These results clearly illustrate that the prediction of stable clusters by the SAO model is well consistent with the DFT calculations.

In the SAO model, there are some parameters that can control the electronic properties while conserving the structural symmetry. One parameter is the number of constitutive atoms: $E_{4}, E_{10}, E_{20}, E_{35}$, etc. The optimized geometries and molecular orbitals of $\mathrm{Mg}_{4}, \mathrm{Mg}_{10}$, and $\mathrm{Mg}_{20}$ are shown in Fig. 4a (see also Supplementary Figs. 3 and 4 for reference). Their 8,20 , and 40 total valence electrons fill the $1 P\left(T_{2}\right), 1 D(E)$, and $2 P\left(T_{2}\right)$ orbitals, respectively. According to Fig. $4 \mathrm{a}$, when the number of constitutive atoms increases, the jellium orbitals overlap more nuclear charges, and thereby become more stabilized. The sizes of the superatomic orbitals can also be controlled by this method. As the atomicity increases, the gap between the highest occupied molecular 

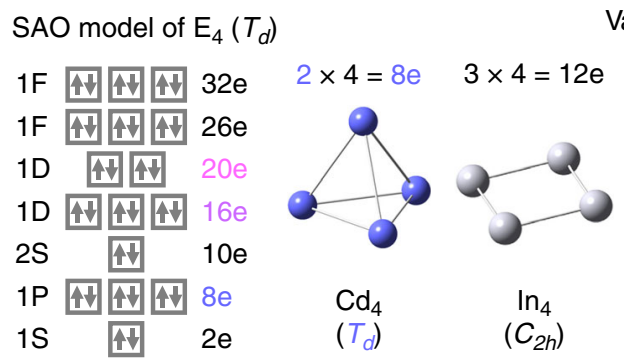

Valence electrons
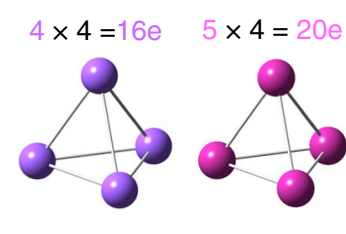

$6 \times 4=24 \mathrm{e}$

Fig. 3 Optimized structures of $E_{4}$ clusters with various valence electrons. The symmetry-adapted orbital (SAO)-based electronic configuration is also shown for reference
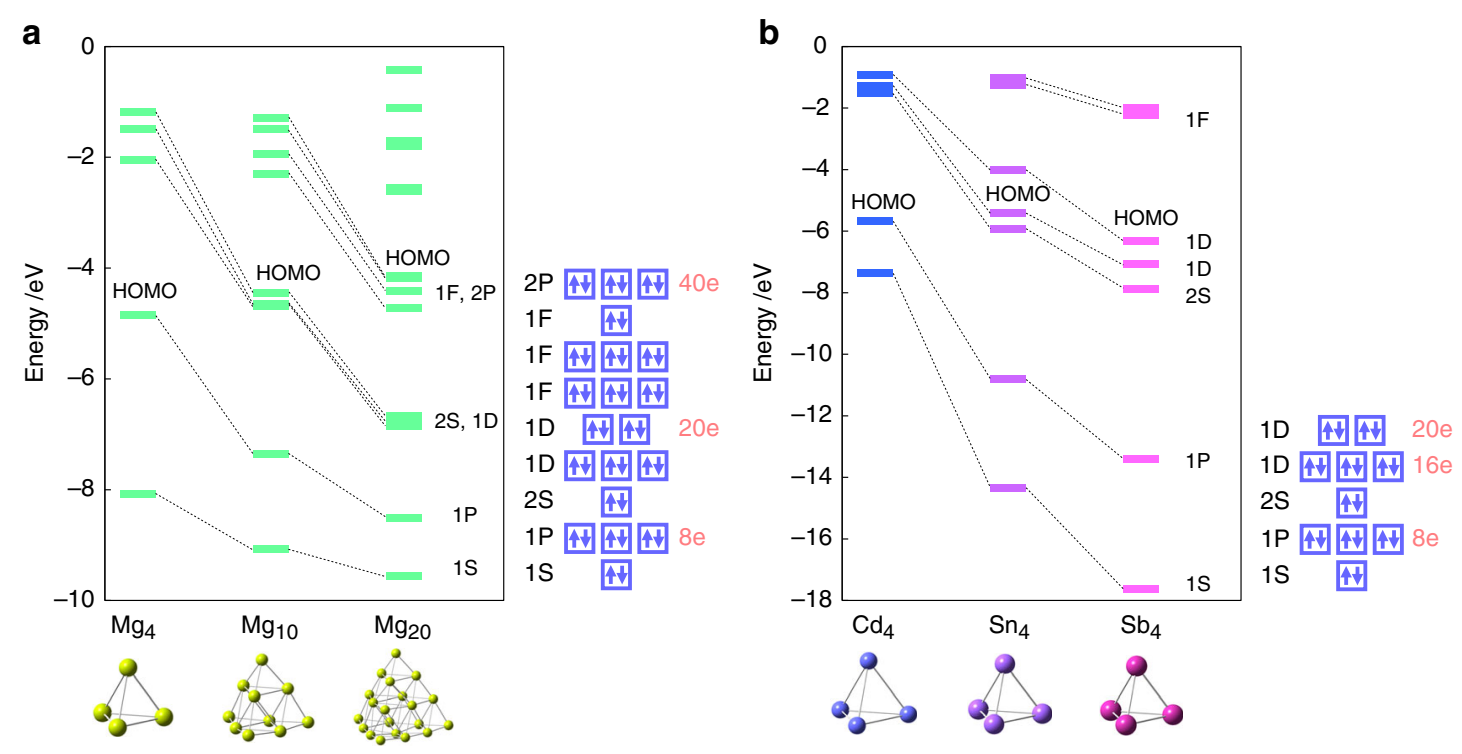

Fig. 4 Orbital levels of different-sized and anisoelectronic clusters. a $\mathrm{Mg}_{4}, \mathrm{Mg}_{10}$, and $\mathrm{Mg}_{20}$. b Cd $\mathrm{Cd}_{4} \mathrm{Sn}_{4}$, and $\mathrm{Sb}_{4}$. The symmetry-adapted orbital (SAO)based electronic configurations are also shown for reference
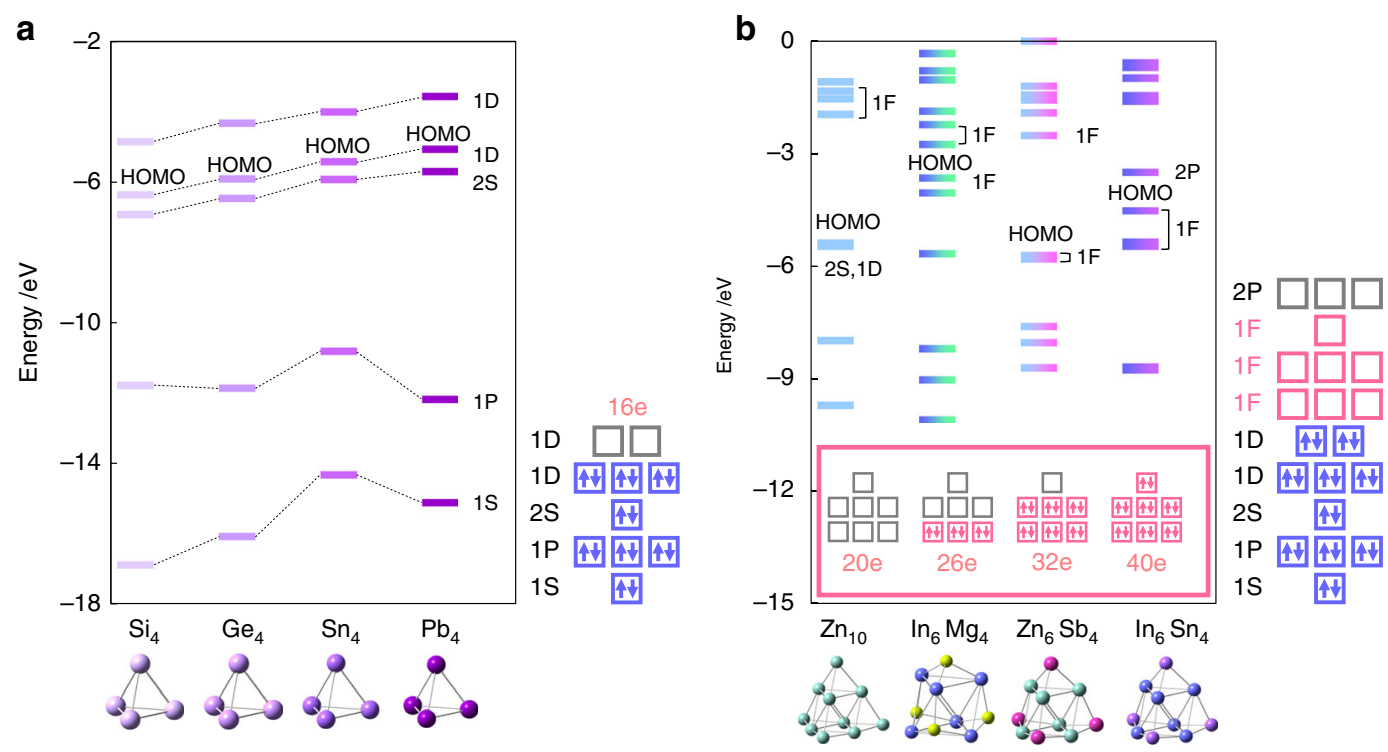

Fig. 5 Orbital levels of isoelectronic and alloy clusters. a $\mathrm{Si}_{4}, \mathrm{Ge}_{4}, \mathrm{Sn}_{4}$, and $\mathrm{Pb}_{4}$. b Zn $\mathrm{Zn}_{10}, \mathrm{In}_{6} \mathrm{Mg}_{4}, \mathrm{Zn}_{6} \mathrm{Sb}_{4}$, and $\ln _{6} \mathrm{Sn}_{4}$. The symmetry-adapted orbital (SAO)based electronic configurations are also shown for reference

orbital (HOMO) and the lowest unoccupied molecular orbital (LUMO) becomes smaller and approaches that of the bulk state.

Another parameter is the constitutive elements. The optimized geometries and molecular orbitals of $\mathrm{Cd}_{4}, \mathrm{Sn}_{4}$, and $\mathrm{Sb}_{4}$, which constitute elements in the same period, are shown in Fig. 4b (see also Supplementary Fig. 5 for reference). When the constitutive elements are different between two clusters, the orbital levels of the clusters are also different, because the effective potentials acting on valence electrons are different. The optimized geometries and molecular orbitals of $\mathrm{Si}_{4}, \mathrm{Ge}_{4}$, 


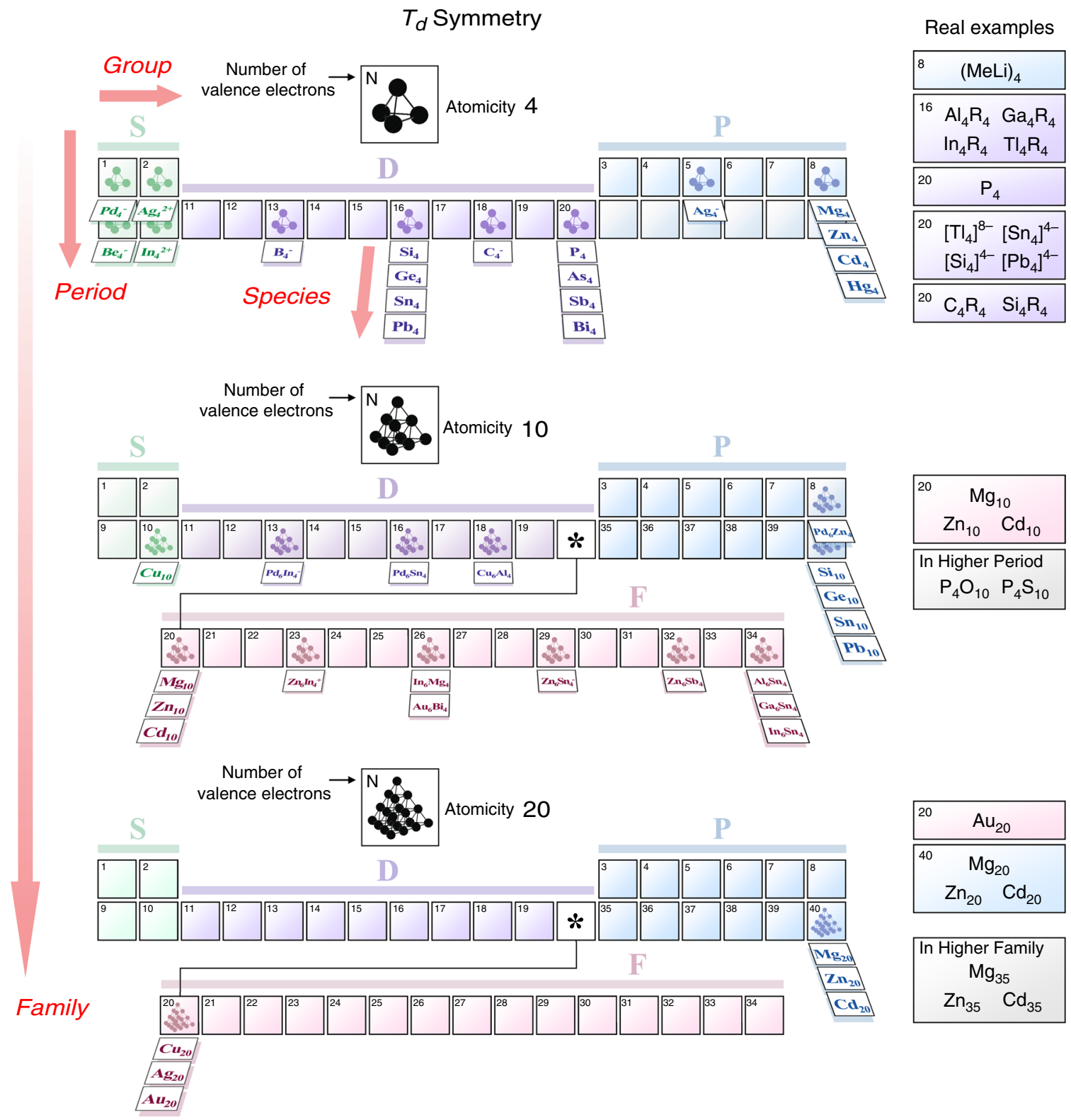

Fig. 6 The periodic framework based on the $T_{d}$-type symmetry-adapted orbital (SAO) model. Groups and periods are defined according to the outer-shell configurations. Families and species classify clusters from the viewpoints of the number of constitutive atoms and the constitutive elements, respectively

$\mathrm{Sn}_{4}$, and $\mathrm{Pb}_{4}$, which are Group 14 elements, are shown in Fig. 5a (see also Supplementary Figs. 6-8 for reference). These elements have 16 valence electrons, which coincide with the same orbital-filling condition. The orbital levels of heavier atoms tend to be less stabilized. The SAO model is also applicable to alloy clusters. Various compositions of elements enable the number of valence electrons to be precisely controlled. Figure $5 \mathrm{~b}$ shows that the number of electrons that occupy the $1 F$ superatomic orbitals can be controlled using alloy clusters. This example is regarded as mimicry of the lanthanide elements. The Pd atom, of which the electronic configuration is $[\mathrm{Kr}] 4 d^{10}$, can be utilized as a dummy element with 0 valence electrons. This technique is also applicable for control of the number of valence electrons (Supplementary Figs. 9 and 10). All of these examples possess $T_{d}$ symmetry without the Jahn-Teller or pseudo Jahn-Teller instability ${ }^{16}$. These computational results thus validate the SAO model.

\section{Discussion}

The calculated clusters can be classified in a periodic framework, in analogy to the periodic table of the elements (Fig. 6). Each piece in the framework can be regarded as an "element". This framework not only has "groups" and "periods" but also "families" and "species" as new dimensions. In a similar way to the conventional periodic table, the groups and periods are defined according to the outer-shell configurations ( $\left(\mathrm{see} \mathrm{Cd}_{4}, \mathrm{Sn}_{4}\right.$, and $\mathrm{Sb}_{4}$ as examples). Thus, there are $S_{-}, P_{-}, D_{-}$, and $F$-blocks. Further, families classify clusters with the same structural symmetry from the viewpoint of the number of constitutive atoms. For example, $\mathrm{Cu}_{10}, \mathrm{Mg}_{10}$, and $\mathrm{Ge}_{10}$ belong to the same family $\mathrm{X}_{10}$, whereas $\mathrm{Mg}_{4}, \mathrm{Mg}_{10}$, and $\mathrm{Mg}_{20}$ belong to different families $\mathrm{X}_{4}, \mathrm{X}_{10}$, and $\mathrm{X}_{20}$, respectively. Species classify clusters with the same structure and the same total number of valence electrons from the viewpoint of the constitutive elements. For example, $\mathrm{Si}_{10}, \mathrm{Ge}_{10}, \mathrm{Sn}_{10}$, and $\mathrm{Pb}_{10}$ are all tetrahedral with 40 valence electrons but belong to different species. In this way, the SAO model directs a spotlight on 
a

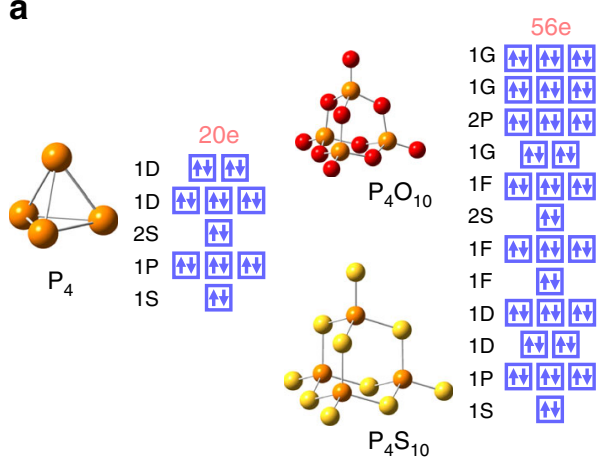

b

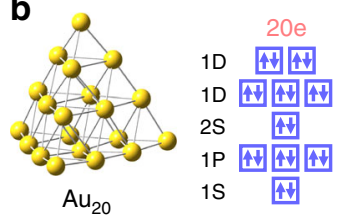

c

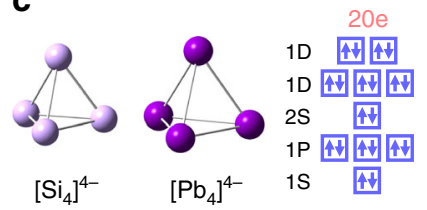

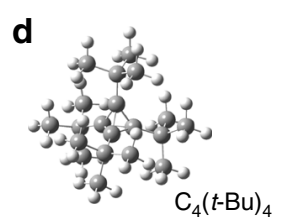
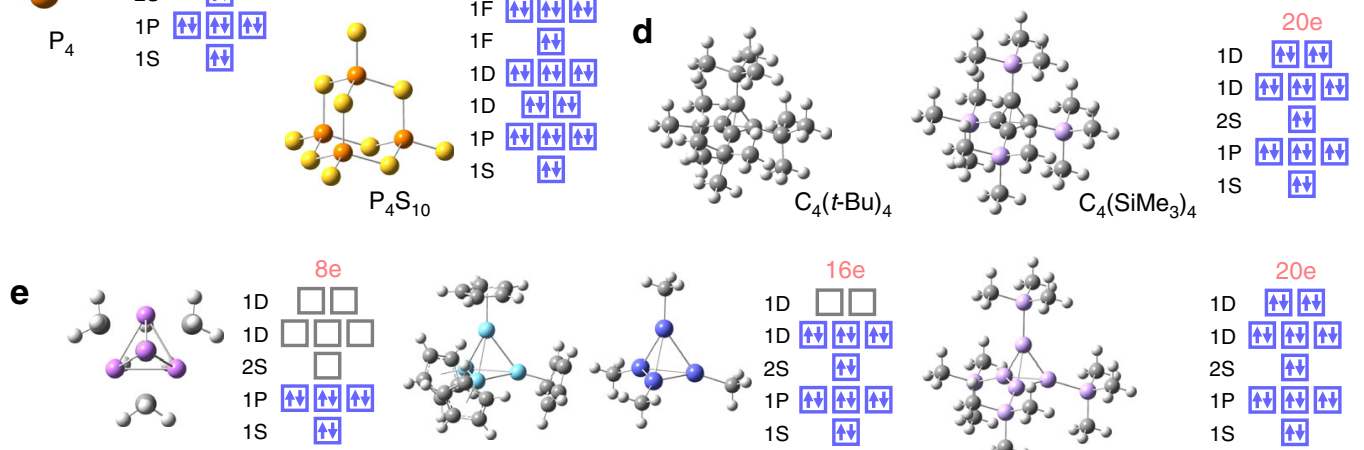

$(\mathrm{MeLi})_{4}$
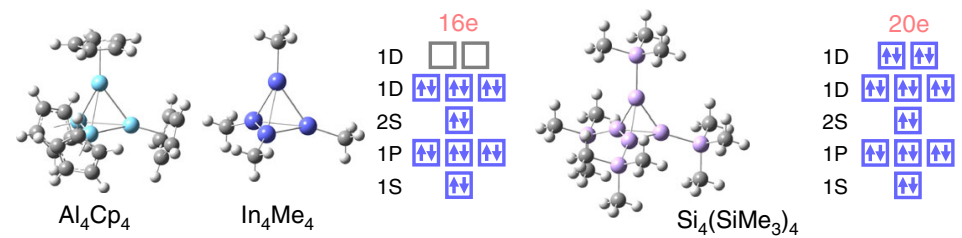

Fig. 7 Electronic configurations of real compounds. a Natural products $\left(\mathrm{P}_{4}, \mathrm{P}_{4} \mathrm{O}_{10}\right.$, and $\left.\mathrm{P}_{4} \mathrm{~S}_{10}\right)$. b The magic gold cluster $\left(\mathrm{Au}_{20}\right)$. c $\mathrm{Zintl}$ anions $\left(\left[\mathrm{Si}_{4}\right]^{4-}\right.$ and $\left.\left[\mathrm{Pb}_{4}\right]^{4-}\right)$. d Organic compounds $\left(\mathrm{C}_{4}(t-\mathrm{Bu})_{4}\right.$ and $\left.\mathrm{C}_{4}\left(\mathrm{SiMe}_{3}\right)_{4}\right)$. e Organometallic clusters $\left((\mathrm{MeLi})_{4}, \mathrm{Al}_{4} \mathrm{Cp}_{4}, \ln _{4} \mathrm{Me}_{4}\right.$, and $\left.\mathrm{Si}_{4}\left(\mathrm{SiMe}_{3}\right)_{4}\right)$

the multi-dimensional periodicity behind an abundance of clusters.

Many real compounds with $T_{d}$ symmetry also support the SAO model. Figure $7 \mathrm{a}$ shows natural white phosphorus $\left(\mathrm{P}_{4}\right)^{20}$ with its pentoxide and pentasulfide $\left(\mathrm{P}_{4} \mathrm{O}_{10} \text { and } \mathrm{P}_{4} \mathrm{~S}_{10}\right)^{21}$. Figure $7 \mathrm{~b}$ shows the famous gold cluster $\left(\mathrm{Au}_{20}\right)^{8}$. All of these substances fulfill the orbital-filling conditions of the SAO model. As for $\mathrm{P}_{4} \mathrm{O}_{10}$ and $\mathrm{P}_{4} \mathrm{~S}_{10}$, their central frameworks $\left(\mathrm{P}_{4} \mathrm{O}_{6}\right.$ and $\left.\mathrm{P}_{4} \mathrm{~S}_{6}\right)$ were used to count the number of valence electrons (see Supplementary Fig. 11). In the gas phase, the $\mathrm{Mg}_{N}, \mathrm{Zn}_{N}$, and $\mathrm{Cd}_{N}(N=10$, 20, and 35) clusters have been detected using mass spectrometry $22-25$. These atomicities are suitable only for the construction of $T_{d}$ symmetric structures and the number of valence electrons coincides with the orbital-filling conditions of the SAO model (Fig. 4 and Supplementary Fig. 4). Figure 7c shows Zintl anions ${ }^{26-28}$ that fit the SAO model, e.g., $\left[\mathrm{Si}_{4}\right]^{4-},\left[\mathrm{Sn}_{4}\right]^{4-},\left[\mathrm{Pb}_{4}\right]^{4-}$, and $\left[\mathrm{Tl}_{4}\right]^{8-}$ (see also Supplementary Fig. 12). Some organic compounds such as tetrahedranes 29,30 also satisfy the SAO model (Fig. 7d and Supplementary Fig. 13) due to one-electron donation from each substituent. Organometallic clusters also follow the SAO model. The alkyllithium tetramer $(\mathrm{RLi})_{4}{ }^{31}$ is a good example of the SAO model (Fig. 7e and Supplementary Fig. 14); it has a total of eight electrons consistent with the SAO model. The stabilities of $\mathrm{M}_{4} \mathrm{R}_{4}(\mathrm{M}=\mathrm{Al}, \mathrm{Ga} \text {, In, and } \mathrm{Tl})^{32,33}$ can be justified with the SAO model (Fig. 7e and Supplementary Fig. 15). Finally, tetrahedro-tetrasilane is also governed by the SAO model ${ }^{34}$ (Fig. 7e).

The SAO model can be applied to the $O_{h}$ and $I_{h}$ symmetries in the same way as that for the $T_{d}$ symmetry. This means that various similar frameworks exist, depending on the symmetry of the clusters (Supplementary Figs. 16 and 17). We herein should note that highly symmetric clusters typified by $I_{h}$ have fewer examples than tetrahedral clusters. This is because it is hard to satisfy the magic-number requirement of valence electrons, as well as that of constitutive atoms simultaneously. For example, it can be easily imagined that the number of valence electrons in a 13-atom monoelemental icosahedral cluster is limited to be multiples of 13 . On the other hand, it should also be noted that there is more than one structural framework belonging to each point group. For instance, both the octahedron and cuboctahedron have the same $O_{h}$ symmetry. The investigation of frameworks other than the present cases will thus lead to the discovery of novel periodic classifications of clusters.

Various innovative materials could be synthesized based on the SAO model (Supplementary Figs. 18 and 19). If the number of occupying electrons matches the degree of the degeneracy of the high-lying SAOs, then a spin arrangement is induced by exchange interactions, without Jahn-Teller instability ${ }^{16}$. In fact, $\left[\mathrm{Na}_{55}\right]^{+}$has been experimentally reported to possess subshell high spins ${ }^{35}$. Magnetic clusters with only nonmagnetic elements could be designed and classified in the $\mathrm{D}$ block, e.g., $\mathrm{Cu}_{6} \mathrm{Al}_{4}$ (Supplementary Fig. 20). We surmise that the lightest magnetic compounds without heavy $d$ elements will be predicted by this approach. On the other hand, clusters classified in the F-blocks could behave as a photofunctional materials similar to the lanthanides (Fig. 5b and Supplementary Fig. 21).

A variety of clusters predicted by the SAO model are expected to be actually synthesized. Laser vaporization techniques combined with time-of-flight mass spectrometry produce many of them in the gas phase. To use the produced clusters as materials, they could be soft-landed onto selfassembled monolayers ${ }^{36}$. In the liquid phase, template-based synthetic methods are useful for the fabrication of clusters ${ }^{37}$. The isolation and crystallization of clusters could be realized by ligand protection ${ }^{38}$. Crystallization techniques have been established for the preparation of Zintl-type clusters ${ }^{39}$. The cocrystallization method for clusters and fullerenes would also be effective to obtain solid-state materials ${ }^{5}$.

In summary, we have proposed a symmetry-adapted orbital model that is beneficial for the prediction and design of stable clusters. This model indicates that an abundance of stable clusters could be classified as elements in a periodic framework, with respect to each structural symmetry. The periodic framework is strongly supported by many quantum chemical calculations and various existing substances. It is not only conceptual but also practical for the systematic exploration of unknown stable clusters. Among the infinite combinations of constitutive elements, this approach will be a significant contribution to the formation of innovative materials based on clusters with magnetic, optical, and catalytic functions. 


\section{Methods}

First-principles calculations. Geometry optimizations and vibrational analyses were conducted using DFT calculations implemented in the Gaussian 09 package, Revision E.01 ${ }^{40}$. The B3LYP functional and LanL2DZ basis set were employed. The 6-31G $(\mathrm{d}, \mathrm{p})$ basis set was used only for $\mathrm{H}, \mathrm{Li}$, and C. Typical elements with $s$ and $p$ valence electrons in their outermost shell are known to be suitable for the formation of superatomic orbitals. The $T_{d}$ symmetric clusters comprised of typical elements were calculated with respect to the various atomicities $\left(E_{4}, E_{10}\right.$, and $\left.E_{20}\right)$. The $s$ - and p-type valence electrons of the elements were counted as 1 for $\mathrm{Li}, \mathrm{Cu}$, $\mathrm{Ag}$, and $\mathrm{Au}, 2$ for $\mathrm{Be}, \mathrm{Mg}, \mathrm{Zn}, \mathrm{Cd}$, and $\mathrm{Hg}, 3$ for $\mathrm{B}, \mathrm{Al}, \mathrm{Ga}$, In, and $\mathrm{Tl}, 4$ for $\mathrm{C}, \mathrm{Si}$, $\mathrm{Ge}, \mathrm{Sn}$, and $\mathrm{Pb}, 5$ for $\mathrm{P}, \mathrm{As}, \mathrm{Sb}$, and $\mathrm{Bi}, 6$ for $\mathrm{O}, \mathrm{S}$, Se, and Te, 7 for I, and 0 for Pd. It was confirmed that all the obtained structures have no imaginary frequencies. A small part of the present clusters has also been discussed in our recent paper ${ }^{41}$, including $\mathrm{Mg}_{4}, \mathrm{Mg}_{10}, \mathrm{Mg}_{20}, \mathrm{Zn}_{4}, \mathrm{Zn}_{10}, \mathrm{Zn}_{20}, \mathrm{Cd}_{4}, \mathrm{Cd}_{10}, \mathrm{Cd}_{20}, \mathrm{Si}_{10}, \mathrm{Ge}_{10}, \mathrm{Sn}_{10}, \mathrm{~Pb}_{10}$ $\mathrm{Al}_{6} \mathrm{Sn}_{4}, \mathrm{Ga}_{6} \mathrm{Sn}_{4}$, and $\mathrm{In}_{6} \mathrm{Sn}_{4}$

\section{Data availability}

The data that support the findings of this study are available from the corresponding author upon reasonable request.

\section{Code availability}

The Gaussian 09 package, Revision E. $01^{40}$ was employed for all the electronic state calculations. The software and instructions for its use are available at http://gaussian. $\mathrm{com} /$.

Received: 15 February 2019 Accepted: 12 July 2019

Published online: 19 August 2019

\section{References}

1. Thyssen, P. \& Ceulemans, A. J. Shattered Symmetry: Group Theory from the Eightfold Way to the Periodic Table. (Oxford University Press, Oxford, 2017).

2. Tomalia, D. A. \& Khanna, S. N. A systematic framework and nanoperiodic concept for unifying nanoscience: hard/soft nanoelements, superatoms, meta-atoms, new emerging properties, periodic property patterns, and predictive Mendeleev-like nanoperiodic tables. Chem. Rev. 116, 2705-2774 (2016)

3. Claridge, S. A. et al. Cluster-assembled materials. ACS Nano 3, 244-255 (2009).

4. Reber, A. C., Khanna, S. N. \& Castleman, A. W. Jr. Superatom compounds clusters, and assemblies: ultra alkali motifs and architectures. J. Am. Chem. Soc. 129, 10189-10194 (2007).

5. Pinkard, A., Champsaur, A. M. \& Roy, X. Molecular clusters: nanoscale building blocks for solid-state materials. Acc. Chem. Res. 51, 919-929 (2018).

6. Bergeron, D. E., Castleman, A. W. Jr., Morisato, T. \& Khanna, S. N. Formation of $\mathrm{Al}_{13} \mathrm{I}^{-}$: evidence for the superhalogen character of $\mathrm{Al}_{13}$. Science 304, 84-87 (2004).

7. Reveles, J. U., Khanna, S. N., Roach, P. J. \& Castleman, A. W. Jr. Multiple valence superatoms. Proc. Natl Acad. Sci. USA 103, 18405-18410 (2006).

8. Li, J., Li, X., Zhai, H. -J. \& Wang, L. -S. $\mathrm{Au}_{20}$ : a tetrahedral cluster. Science 299 864-867 (2003).

9. Reveles, J. U. et al. Designer magnetic superatoms. Nat. Chem. 1, 310-315 (2009).

10. Weigend, F. \& Ahlrichs, R. Quantum chemical treatments of metal clusters Philos. Trans. R. Soc. A 368, 1245-1263 (2010)

11. Jena, P. Beyond the periodic table of elements: the role of superatoms. J. Phys. Chem. Lett. 4, 1432-1442 (2013).

12. Xu, W. W., Zhu, B., Zeng, X. C. \& Gao, Y. A grand unified model for liganded gold clusters. Nat. Commun. 7, 1-8 (2016)

13. de Heer, W. A. The physics of simple metal clusters: experimental aspects and simple models. Rev. Mod. Phys. 65, 611-676 (1993).

14. Piotrowski, M. J., Piquini, P. \& Da Silva, J. L. F. Density functional theory investigation of $3 d, 4 d$, and $5 d$ 13-atom metal clusters. Phys. Rev. B 81, 1-14 (2010).

15. Chaves, A. S., Piotrowski, M. J. \& Da Silva, J. L. F. Evolution of the structural, energetic, and electronic properties of the $3 \mathrm{~d}, 4 \mathrm{~d}$, and $5 \mathrm{~d}$ transition-metal clusters $\left(30 \mathrm{TM}_{n}\right.$ systems for $\left.n=2-15\right)$ : a density functional theory investigation. Phys. Chem. Chem. Phys. 19, 15484-15502 (2017).

16. Bersuker, I. B. The Jahn-Teller Effect. (Cambridge University Press, Cambridge, 2006).

17. Altmann, S. L. \& Herzig, P. Point-Group Theory Tables, 2nd edn. (Clarendon Press, Wien, 2011)
18. Ceulemans, A. J. Theoretical Chemistry and Computational Modelling. Group Theory Applied to Chemistry (Springer, Dordrecht, 2013).

19. Medel, V. M. et al. Hund's rule in superatoms with transition metal impurities. Proc. Natl Acad. Sci. USA 108, 10062-10066 (2011).

20. Housecroft, C. \& Sharpe, A. G. Inorganic Chemistry, 4th edn. (Pearson Prentice Hall, Upper Saddle River, 2012).

21. Corbridge, D. E. C. Phosphorus. An Outline of Its Chemistry, Biochemistry, and Technology. 2nd edn (Elsevier Scientific Co., Amsterdam, 1980).

22. Katakuse, I. et al. Correlation between mass distributions of zinc, cadmium clusters and electronic shell structure. Int. J. Mass Spectrom. Ion Processes 69, 109-114 (1986).

23. Katakuse, I. et al. Mass distributions of positive and negative cluster ions of zinc and cadmium. Int. J. Mass Spectrom. Ion Processes 91, 85-91 (1989).

24. Diederich, T., Döppner, T., Braune, J., Tiggesbäumker, J. \& Meiwes-Broer, K. -H. Electron delocalization in magnesium clusters grown in supercold helium droplets. Phys. Rev. Lett. 86, 4807-4810 (2001).

25. Diederich, T., Döppner, T., Fennel, T., Tiggesbäumker, J. \& Meiwes-Broer, K. -H. Shell structure of magnesium and other divalent metal clusters . Phys. Rev. A. 72, 1-11 (2005).

26. Lorenz, C., Gärtner, S. \& Korber, N. $\mathrm{Si}_{4}^{4-}$ in solution-first solvate crystal structure of the ligand-free tetrasilicide tetraanion in $\mathrm{Rb}_{1.2} \mathrm{~K}_{2.8} \mathrm{Si}_{4} \cdot 7 \mathrm{NH}_{3}$. $\mathrm{Z}$. Anorg. Allg. Chem. 643, 141-145 (2017).

27. Wiesler, K., Brandl, K., Fleischmann, A. \& Korber, N. Tetrahedral $\left[\mathrm{Tt}_{4}\right]^{4-}$ zintl anions through solution chemistry: syntheses and crystal structures of the ammoniates $\mathrm{Rb}_{4} \mathrm{Sn}_{4} \cdot 2 \mathrm{NH}_{3}, \mathrm{Cs}_{4} \mathrm{Sn}_{4} \cdot 2 \mathrm{NH}_{3}$, and $\mathrm{Rb}_{4} \mathrm{~Pb}_{4} \cdot 2 \mathrm{NH}_{3}$. Z. Anorg. Allg. Chem. 635, 508-512 (2009).

28. Dong, Z. -C. \& Corbett, J. D. $\mathrm{Na}_{23} \mathrm{~K}_{9} \mathrm{Tl}_{15.3}$ : an unusual zintl compound containing apparent $\mathrm{Tl}_{5}^{7-}, \mathrm{Tl}_{4}^{8-}, \mathrm{Tl}_{3}^{7-}$, and $\mathrm{Tl}^{5-}$ anions. Inorg. Chem. 35, 3107-3112 (1996)

29. Maier, G., Pfriem, S., Schäfer, U. \& Matusch, R. Tetra- tert-butyltetrahedrane. Angew. Chem. Int. Ed. Engl. 17, 520-521 (1978).

30. Maier, G. et al. Tetrakis(trimethylsilyl)tetrahedrane. J. Am. Chem. Soc. 124, 13819-13826 (2002)

31. Elschenbroich, C. Organometallics, 5th edn. (Wiley-VCH Verlag GmbH \& Co. KGaA, Weinheim, 2016).

32. Dohmeier, C., Loos, D. \& Schnöckel, H. Aluminum(I) and gallium(I) compounds: syntheses, structures, and reactions. Angew. Chem. Int. Ed. Engl. 35, 129-149 (1996)

33. Uhl, W. Tetrahedral homonuclear organoelement clusters and subhalides of aluminium, gallium and indium. Naturwissenschaften 91, 305-319 (2004).

34. Wiberg, N., Finger, C. M. M. \& Polborn, K. Tetrakis(tri- tert-butylsilyl)tetrahedro-tetrasilane $\left(t \mathrm{Bu}_{3} \mathrm{Si}_{4}\right)_{4} \mathrm{Si}_{4}$ : the first molecular silicon compound with a $\mathrm{Si}_{4}$ tetrahedron. Angew. Chem. Int. Ed. Engl. 32, 1054-1056 (1993).

35. Vásquez-Pérez, J. M. et al. Influence of spin multiplicity on the melting of $\mathrm{Na}_{55}{ }^{+}$. J. Phys. Chem. Lett. 6, 4646-4652 (2015).

36. Mitsui, M., Nagaoka, S., Matsumoto, T. \& Nakajima, A. Soft-landing isolation of vanadium-benzene sandwich clusters on a room-temperature substrate using $n$-alkanethiolate self-assembled monolayer matrixes. J. Phys. Chem. B 110, 2968-2971 (2006).

37. Crooks, R. M., Zhao, M., Sun, L., Chechik, V. \& Yeung, L. K. Dendrimerencapsulated metal nanoparticles: synthesis, characterization, and applications to catalysis. Acc. Chem. Res. 34, 181-190 (2001)

38. Walter, M. et al. A unified view of ligand-protected gold clusters as superatom complexes. Proc. Natl Acad. Sci. USA 105, 9157-9162 (2008).

39. Scharfe, S., Kraus, F., Stegmaier, S., Schier, A. \& Fässler, T. F. Zintl ions, cage compounds, and intermetalloid clusters of group 14 and group 15 elements. Angew. Chem. Int. Ed. 50, 3630-3670 (2011).

40. Frisch, M. J. et al. Gaussian 09 Revision E.01 (Gaussian Inc., Wallingford, CT, 2015).

41. Haruta, N., Tsukamoto, T., Kuzume, A., Kambe, T. \& Yamamoto, K. Nanomaterials design for super-degenerate electronic state beyond the limit of geometrical symmetry. Nat. Commun. 9, 3758 (2018)

\section{Acknowledgements}

This study was supported by a grant from JST ERATO Grant Number JPMJER1503, Japan (K. Y.), JSPS KAKENHI Grant Number JP19K15583 (T. T.), and JSPS KAKENHI Grant Number JP19K15501 (N. H.).

\section{Author contributions}

T.T. and N.H. initiated and contributed equally to this work. K.Y. supervised it. T.T. and N.H. constructed the SAO model, conducted the DFT calculations, and analyzed the results of the calculations. T.T., N.H., T.K., A.K. and K.Y. participated in the discussion and writing of the manuscript. 


\section{Additional information}

Supplementary Information accompanies this paper at https://doi.org/10.1038/s41467019-11649-0.

Competing interests: The authors declare no competing interests.

Reprints and permission information is available online at http://npg.nature.com/ reprintsandpermissions/

Peer review information: Nature Communications thanks Shiv Khanna, Arnout Ceulemans and other anonymous reviewer(s) for their contribution to the peer review of this work

Publisher's note: Springer Nature remains neutral with regard to jurisdictional claims in published maps and institutional affiliations. (c) (i) Open Access This article is licensed under a Creative Commons Attribution 4.0 International License, which permits use, sharing, adaptation, distribution and reproduction in any medium or format, as long as you give appropriate credit to the original author(s) and the source, provide a link to the Creative Commons license, and indicate if changes were made. The images or other third party material in this article are included in the article's Creative Commons license, unless indicated otherwise in a credit line to the material. If material is not included in the article's Creative Commons license and your intended use is not permitted by statutory regulation or exceeds the permitted use, you will need to obtain permission directly from the copyright holder. To view a copy of this license, visit http://creativecommons.org/ licenses/by/4.0/.

(C) The Author(s) 2019 